\title{
Navigation-guided transsylvian approach and microsurgical resection of a deep left temporal low-grade arteriovenous malformation
}

\author{
William T. Couldwell, MD, PhD \\ Department of Neurosurgery, Clinical Neurosciences Center, University of Utah, Salt Lake City, Utah
}

This video demonstrates stereotactic-guided resection of a ruptured diffuse left temporal arteriovenous malformation (AVM) in an adolescent male who presented with headache and speech difficulties. The diffuse nidus of the AVM, 25 $\mathrm{mm}$ in size, was located in the posterior superior temporal gyrus, with drainage into the sylvian veins (Spetzler-Martin Grade II). The AVM was located stereotactically, and resection was performed through a small corticectomy. The clot cavity was evacuated. Feeding branches to the AVM were identified during careful dissection, and parent M1 and M2 branches were preserved. The patient recovered well, with no residual speech deficit. Postoperative angiogram demonstrated complete AVM removal.

The video can be found here: https://youtu.be/Sttc86H8jCw.

KEYWORDS AVM; arteriovenous malformation; ruptured; stereotactic guidance; resection 